\title{
氮化硼气凝胶的制备及其应用进展
}

\author{
柳凤琦，冯坚，姜勇刚，李良军 \\ (国防科技大学 新型陶瓷纤维及其复合材料国防科技重点实验室, 长沙 410073)
}

\begin{abstract}
摘 要: 氮化硼气凝胶是一类以固体为骨架、气体为分散介质的, 具有三维多孔网络结构的新型纳米材料, 展现出 高比表面积、高孔隙率、低密度等优异的性能。此外, 相比于石墨烯气凝胶, 氮化硼气凝胶拥有更好的绝缘性、抗 氧化性、热稳定性和化学稳定性, 因此它在气体吸附、催化、污水净化、导热/隔热等领域极具应用前景。本文结 合国内外研究现状, 重点介绍了硬模板法、软模板法、低维氮化嗍组装法和无模板法制备氮化嗍气凝胶的结构和性 能特点，总结了其在关键领域的重要应用，并对其未来发展方向进行了展望。
\end{abstract}

关 键 词: 氮化嗍气凝胶; 吸附; 催化; 导热; 隔热; 综述

中图分类号: TQ174 文献标识码: A

\section{Preparation and Application of Boron Nitride Aerogels}

\section{LIU Fengqi, FENG Jian, JIANG Yonggang, LI Liangjun}

(Science and Technology on Advanced Ceramic Fibers and Composites Laboratory, College of Aerospace Science and Engineering, National University of Defense Technology, Changsha 410073, China)

\begin{abstract}
Boron nitride aerogel is a kind of new nanomaterials with three-dimensional porous network structure, which takes solid as the framework and gas as the dispersion medium. It has high specific surface area, high porosity, low density and other excellent properties. In addition, compared with graphene aerogels, it exhibits better insulation, oxidation resistance, thermal stability and chemical stability. These outstanding properties make it promising application in the fields of gas adsorption, catalysis, sewage purification, thermal insulation/conduction. This article systematically reviewed the preparation methods of boron nitride aerogels including the hard template method, soft template method, low-dimensional boron nitride assembly method, and template-free method in the light of domestic and foreign research status. Moreover, the important applications of boron nitride aerogels in key fields are summarized, and the future development direction is prospected.
\end{abstract}

Key words: boron nitride aerogels; adsorption; catalysis; thermal conductivity; thermal insulation; review

氮化嗍(Boron nitride, $\mathrm{BN}$ ) 是由等数量硼原子和 氮原子构成的一种性能优异、极具发展前景的宽带 隙材料 ${ }^{[1]}$, 其晶体类型主要包括三种： $\mathrm{sp}^{2}$ 杂化的六 方氮化嗍 $(\mathrm{h}-\mathrm{BN})^{[2]}, \mathrm{sp}^{3}$ 杂化的立方氮化硼 $(\mathrm{c}-\mathrm{BN})$ 和 纤锌矿型氮化硼 $(\mathrm{w}-\mathrm{BN})^{[3-4]}$ 。其中六方氮化嗍(h-BN)
与石墨具有相似的层状结构和晶格参数, 并且在室 温下呈象牙白色，故也被称作“白石墨”,[5]，其层内 是由交替排列的 $\mathrm{N}$ 原子和 $\mathrm{B}$ 原子以 $\mathrm{sp}^{2}$ 杂化轨道形 成的六角型蜂巢结构二维网络, 层间由微弱的范德 华力结合, 相邻层的六元环属于 $\mathrm{AA}^{\prime}$ 堆积, 并且 $\mathrm{B}$

收稿日期: 2019-12-11; 收到修改稿日期：2020-01-12

基金项目: 湖南省自然科学基金(2018JJ2469) Natural Science Foundation of Hunan Province (2018JJ2469)

作者简介: 柳凤琦(1995-), 男, 博士研究生. E-mail: nudtliufengqi@163.com LIU Fengqi, male, PhD candidate. E-mail: nudtliufengqi@163.com

通讯作者: 冯 坚, 教授. E-mail: fengj@nudt.edu.cn FENG Jian, professor. E-mail: fengj@nudt.edu.cn 
原子与 $\mathrm{N}$ 原子延 $C$ 轴依次交替排列(如图 1) ${ }^{[6]}$ 。六 方氮化嗍 $(\mathrm{h}-\mathrm{BN})$ 与石墨互为等电子体 ${ }^{[7]}$, 因此二者 具有相似的性质, 如高热导率、低摩擦系数、低热 膨胀系数、良好的热力学和化学稳定性等 ${ }^{[8-10]}$ 。近 年来, 鉴于石墨烯(Graphene, G) 材料优异的性能及 其潜在的应用价值, 其研究与应用开发持续升温。 作为石墨烯的类似体, BN 纳米材料也逐渐得到研究 者的青睐, 在能源、催化、传感、生物医学等领域 已取得了一系列重要进展 ${ }^{[11-13]}$ 。

气凝胶材料是一类以固体为骨架、气体为分散 介质的具有三维多孔网络结构的新型纳米材料, 凭 借自身低热导 $\left(0.01 \sim 0.04 \mathrm{~W} \cdot \mathrm{m}^{-1} \cdot \mathrm{K}^{-1}\right)$ 、低密度 $(0.003 \sim$ $\left.0.3 \mathrm{~g} \cdot \mathrm{cm}^{3}\right)$ 、高孔隙率 $(80 \% \sim 99.8 \%)$ 、高比表面积 $\left(500 \sim 1300 \mathrm{~m}^{2} \cdot \mathrm{g}^{-1}\right)$ 等独特性能, 已经广泛应用在建

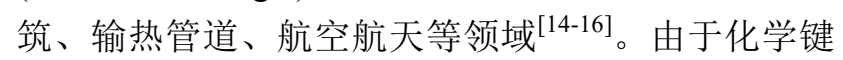
的差异 $(\mathrm{B}-\mathrm{N}$ 键与 $\mathrm{C}=\mathrm{C}$ 键 $), \mathrm{BN}$ 气凝胶表现出不同于 石墨烯气凝胶 (Graphene aerogel, GA) 的独特性能。

例如, 相比于 GA, BN 气凝胶具有更强的抗氧化性 和化学情性, 在空气中的抗氧化温度 $\left(\sim 900{ }^{\circ} \mathrm{C}\right)$ 远高 于 $\operatorname{GA}\left(\sim 450{ }^{\circ} \mathrm{C}\right)$, 并且与酸、碱和熔融金属不易发 生化学反应 ${ }^{[17]}$; 另外, 由于 $\mathrm{N}$ 原子的电负性小于 $\mathrm{B}$ 原子, 电子云向 $\mathrm{N}$ 原子偏移并被束缚, 因此共轭的 $\pi$ 电子不能完全离域形成大 $\pi$ 键 $^{[18]}$, 这决定了 $\mathrm{BN}$ 气 凝胶是一种宽带隙的绝缘体 $(5.9 \mathrm{eV})$, 使其在诸多 领域具有更显著的优势及应用价值。本文从近年来 国内外 BN气凝胶的研究进展出发, 系统介绍了 BN 气凝胶的制备方法及其在气体吸附、催化、污水净 化及导热/隔热等领域的应用, 并对其未来发展方向 进行了展望。

\section{BN 气凝胶的制备方法}

随着 $\mathrm{BN}$ 气凝胶得到不同领域研究者的高度关注, 针对 BN气凝胶制备方法的研究也方兴未艾, 现主要

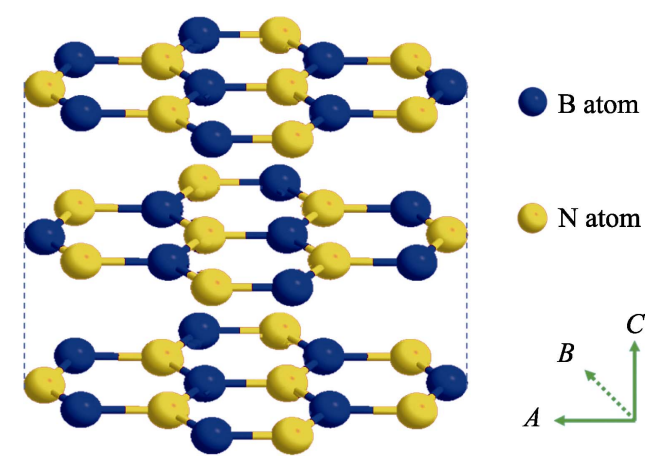

图 1 六方氮化嗍分子结构示意图

Fig. 1 Molecular structure of hexagonal boron nitride
发展成模板法、低维 BN 组装法、无模板法等合成 策略。就模板法而言，根据模板的不同，具体又分为 以活性炭、GA、沸石等多孔材料为模板的硬模板法 和以阳离子表面活性剂、嵌段共聚物等高分子为模 板的软模板法 ${ }^{[19-20]}$ 。模板法是一种发展较为成熟的 制备方法, 但采用硬模板法制备的 BN气凝胶的比表 面积大多在 100 $1100 \mathrm{~m}^{2} \cdot \mathrm{g}^{-1}$ 范围内, 而采用无模板法 制备出的 BN 气凝胶比表面积可高达 $1900 \mathrm{~m}^{2} \cdot \mathrm{g}^{-1[21]}$ 。 相比于其他制备方法, 低维 BN 组装法更适用于大 规模制备 $\mathrm{BN}$ 气凝胶。根据理论计算, 稳定的 $\mathrm{BN}$ 多孔结构的比表面积最大可达 $4800 \mathrm{~m}^{2} \cdot \mathrm{g}^{-1[22-23]}$, 这 表明高比表面积 $\mathrm{BN}$ 气凝胶的制备仍有很大的发展 潜力。

\section{1 硬模板法}

硬模板法是指以多孔无机非金属或金属材料为 模板, 采用化学方法在其上生长 $\mathrm{BN}$, 再采用化学刻 蚀法去除模板后得到与模板具有相似微观形貌的 $\mathrm{BN}$ 气凝胶。2004 年, Han 等 ${ }^{[24]}$ 首次报道了以活性炭 为模板, 以 $\mathrm{B}_{2} \mathrm{O}_{3}$ 和 $\mathrm{N}_{2}$ 为硼源和氮源, 在高温下合成 出多孔 $\mathrm{BN}$ 气凝胶的方法。虽然 $\mathrm{BN}$ 气凝胶继承了 活性炭模板的多孔结构, 但制备出的 BN 气凝胶比 表面积只有 $167.8 \mathrm{~m}^{2} \cdot \mathrm{g}^{-1}$, 远低于活性炭模板的比表 面积 $\left(779 \mathrm{~m}^{2} \cdot \mathrm{g}^{-1}\right)$ (如图 2), 这是由于活性炭的孔径 分布不均, 先驱体在多孔模板中填充不充分导致 的。研究表明, 这种比表面积大幅下降的现象在硬 模板法中普遍存在, 为了克服这一问题, 人们将孔 径分布均匀的多孔材料应用到硬模板法中。Mokaya 等 ${ }^{[25]}$ 在 SBA-15 介孔分子篮模板中渗入 $\mathrm{BH}_{3} \mathrm{NH}_{3}$ 先 驱体制备出比表面积为 $327 \mathrm{~m}^{2} \cdot \mathrm{g}^{-1}$ 的 $\mathrm{BN}$ 气凝胶, 但 较小的比表面积仍制约着 $\mathrm{BN}$ 气凝胶性能的发挥。
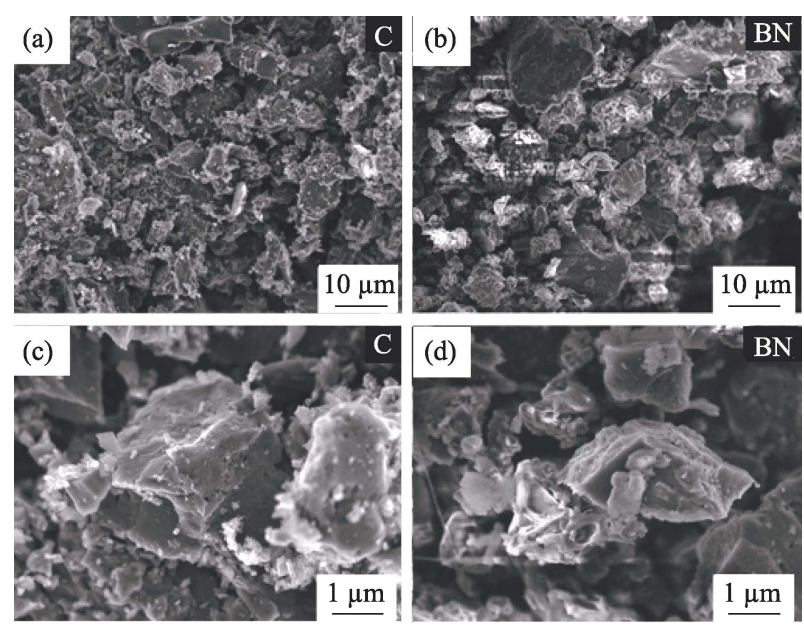

图 $2(\mathrm{a}, \mathrm{b})$ 活性炭和 $(\mathrm{c}, \mathrm{d}) \mathrm{BN}$ 气凝胶的 SEM 照片 ${ }^{[24]}$

Fig. 2 SEM images of $(a, b)$ activated carbon and (c, d) BN aerogels ${ }^{[24]}$ 
近期, $\mathrm{Xu}$ 等 ${ }^{[26-27]}$ 采用冷冻干燥法首先制备出具 有负泊松比和负热膨胀系数的双曲线型多孔 $\mathrm{GA}$ 模 板, 然后在此基础上采用 CVD 法在其上沉积 $\mathrm{BN}$, 经热刻蚀后得到比表面积为 $1080 \mathrm{~m}^{2} \cdot \mathrm{g}^{-1}$ 的 $\mathrm{BN}$ 气凝 胶(如图 3(a))。该气凝胶密度仅为 $0.1 \mathrm{mg} \cdot \mathrm{cm}^{-3}$, 是 目前为止所报道的最轻的固体材料(如图 3(b))。此 外, 负泊松比使 BN 气凝胶具有良好的机械性能和 超弹性, 其相对高度和杨氏模量在 100 次压缩循环 后，仍可以保留 90\%以上(如图 3(c, d))。除了作为生 长模板, 无机非金属模板在高温下还可被 $\mathrm{B}$ 原子和 $\mathrm{N}$ 原子取代, 进而得到 $\mathrm{BN}$ 气凝胶或杂化 $\mathrm{BN}$ 气凝 胶。Kutty 等 ${ }^{[28]}$ 以掺杂碳纳米管的氧化石墨烯(GO) 为模板, 在 $1600{ }^{\circ} \mathrm{C}$ 下通入 $\mathrm{N}_{2}$ 和 $\mathrm{B}_{2} \mathrm{O}_{3}$ 后发生原子取 代反应，成功制得比表面积为 $716.56 \mathrm{~m}^{2} \cdot \mathrm{g}^{-1}$ 的 $\mathrm{BN}$ 气凝胶。另外, 通过改变实验温度等条件, 可以实现 原子不完全取代, 制备出含 $\mathrm{B} 、 \mathrm{~N} 、 \mathrm{C}$ 三种元素的气 凝胶材料。

不同于无机非金属模板，金属模板在反应中同 时还充当着催化剂的角色。Yin 等 ${ }^{[29]}$ 以氨硼烷为先 驱体，在多孔镍泡沫上生长 $\mathrm{BN}$ ，去除模板后得到具 有超低介电常数和超弹性的 $\mathrm{BN}$ 气凝胶, 该气凝胶 仍维持了泡沫镍模板的形貌与结构。另外, 将模板
法与原子取代法相结合可以间接地制备出 $\mathrm{BN}$ 气凝 胶, 例如 Alanzun 等 ${ }^{[30]}$ 首先在硅模板上生长碳泡沫, 进而用 $\mathrm{B}$ 原子和 $\mathrm{N}$ 原子取代 $\mathrm{C}$ 原子得到具有多级孔 结构的 BN 气凝胶。与软模板相比, 硬模板具有较 高的稳定性和良好的空间限域作用，能严格地控制 纳米材料的大小和形貌。但硬模板结构比较单一, 因此用硬模板法制备的纳米材料的形貌通常变化也 较少。

\section{2 软模板法}

软模板通常是由表面活性剂分子聚集而成，主 要包括两亲分子形成的各种有序聚合物，如囊泡、 胶团、微乳液、自组装膜、生物分子和高分子的自组 织结构等 ${ }^{[31-34]}$ 。软模版法是通过分子间的弱相互作 用力引导和调控游离前驱体的规律性组装, 进而形 成具有特异结构纳米材料的方法。Meile 等 ${ }^{[35]}$ 将表面 活性剂十六烷基三甲基溴化铵(CATB)和三甲基环 硼氮烷(MAB)混合后在 $120{ }^{\circ} \mathrm{C}$ 长时间热处理，去除溶 剂后制备出比表面积为 $800 \mathrm{~m}^{2} \cdot \mathrm{g}^{-1}$ 的 $\mathrm{BN}$ 气凝胶(孔 径约为 $6 \mathrm{~nm}$ )。Malenfant 等 ${ }^{[36]}$ 利用有机-无机杂化 的嵌段共聚物聚降冰片烯一癸硼烷(polynorbornenedecaborane), 在四氢呋喃(THF)中自组装制备出多 孔 $\mathrm{BN}$ 气凝胶(图 4, 比表面积为 $950 \mathrm{~m}^{2} \cdot \mathrm{g}^{-1}$ )。软模

(a)

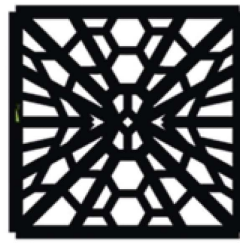

Substructure design Hyperbolic-porous
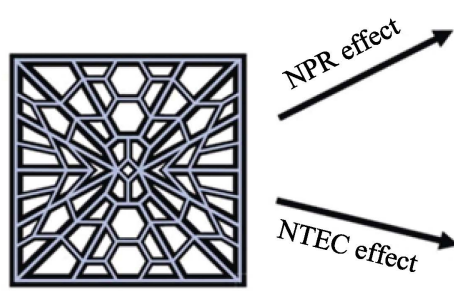

Double-pane-hyperbolic-porous

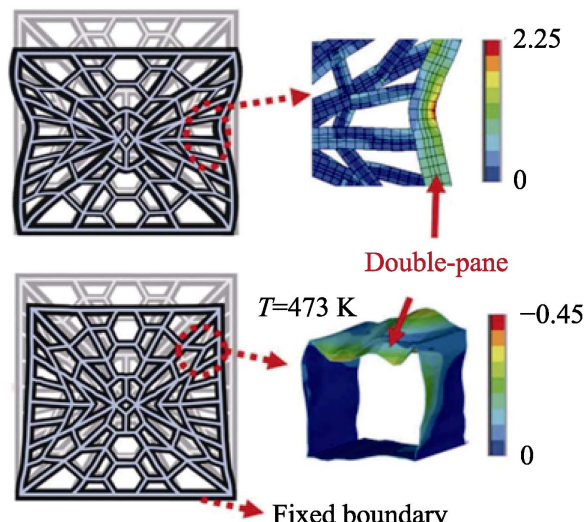

$\rightarrow$ Fixed boundary
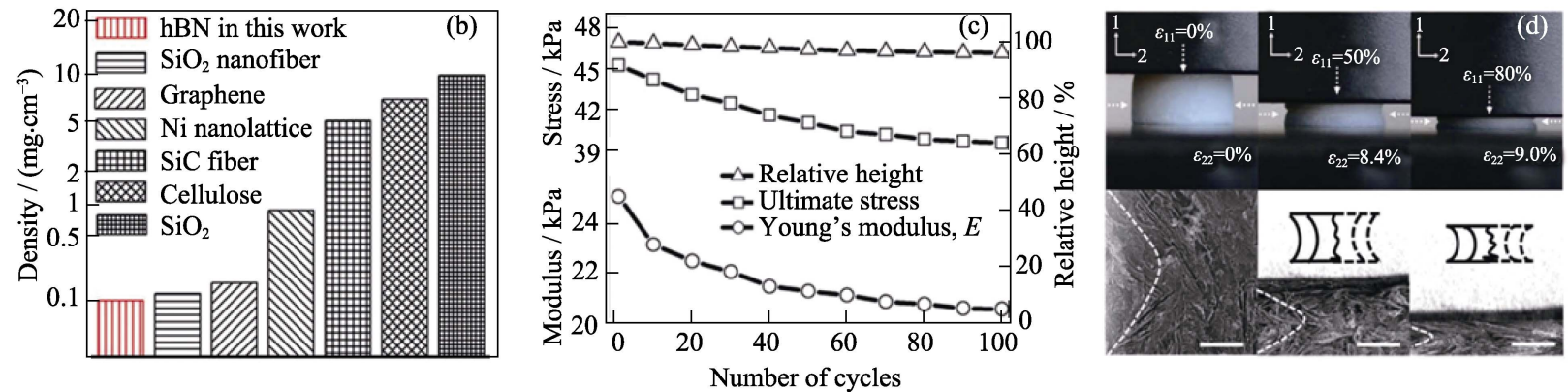

图 3 (a)双壁双曲线型 BN 气凝胶的制备流程示意图, (b)BN 气凝胶与其他材料的密度对比, (c)BN 气凝胶的相对高度、 最大压力和杨氏模量与循环次数的关系曲线, (d)BN 气凝胶在不同压力下的光学照片和 SEM 照片 ${ }^{[26]}$

Fig. 3 (a) Schematic illustration of the metastructure design of BN aerogels; (b) The lightest hBN aerogels sample compared with other ultralight materials; (c) The ultimate stress, Young's modulus, and relative height for 100 compression cycles; (d) Optical and SEM images of BN aerogels under different pressures ${ }^{[26]}$ 


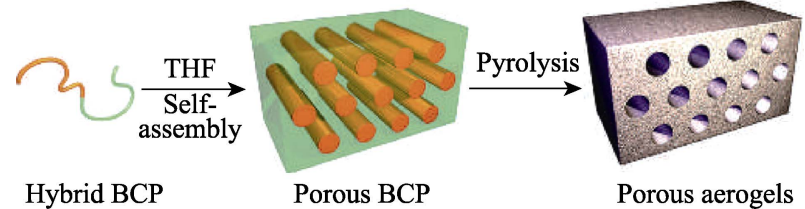

图 4 有机一无机杂化的嵌段共聚物聚降冰片烯一癸硼烷制备 多孔 $\mathrm{BN}$ 流程示意图 ${ }^{[36]}$

Fig. 4 Schematic illustration of organic-inorganic hybrid block copolymer polynorbornene-decorane for preparing $\mathrm{BN}$ aerogels ${ }^{[36]}$

版法难以控制产物的尺寸和形貌，效率较低，但软 模板法具有形态多样、模板易去除、成本低廉等优势。

\section{3 低维 BN 组装法}

低维 BN 组装法是指利用纳米管、纳米带、纳 米片等低维 $\mathrm{BN}$ 纳米材料通过分子桥接、静电相互 作用、范德华力等共价键或非共价键作用, 聚集组

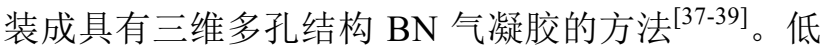
维组装法反应条件较为温和, 并且可以保留低维 $\mathrm{BN}$ 纳米材料的原有特性，以制备具有特殊功能的
BN 气凝胶。2012 年, Jung 等 ${ }^{[40]}$ 发现低维 BN 纳米 材料(包括纳米线、纳米管和纳米片)在胆酸钠中分 散后，经低温蒸发溶剂、溶剂置换和超临界干燥可 组装成具有三维网络结构的 BN 气凝胶(图 5(a))。该 课题组后续又采用类似方法以 $\mathrm{MnO}_{2}$ 纳米线、碳纳 米管、 $\mathrm{MoS}_{2}$ 和石墨烯纳米片为原料制备出多种气凝 胶(图 5(b)), 表明这是一种较为通用的制备气凝胶 的方法。近期， $\mathrm{Li}$ 等 $^{[41]}$ 采用氨化三聚氧胺二硼酸的 方法制备出由纳米带相互缠绕而成的 $\mathrm{BN}$ 气凝胶 (图 6(a))。该气凝胶在-196 1000 ${ }^{\circ} \mathrm{C}$ 范围内仍能够 很好地保持压缩-回弹性能(图 6(b,c)), 在极端环境 中有广阔的应用前景。

随着“绿色化学”的兴起, 研究者们致力于利 用化学技术在化学反应中减少催化剂、产物及副产 物的使用与产生。范德华固体是指二维材料通过范 德华力直接进行堆叠组装, 从而避免了交联剂的使 用。2012 年, GAO 等 ${ }^{[42}$ 通过液相剥离方法制
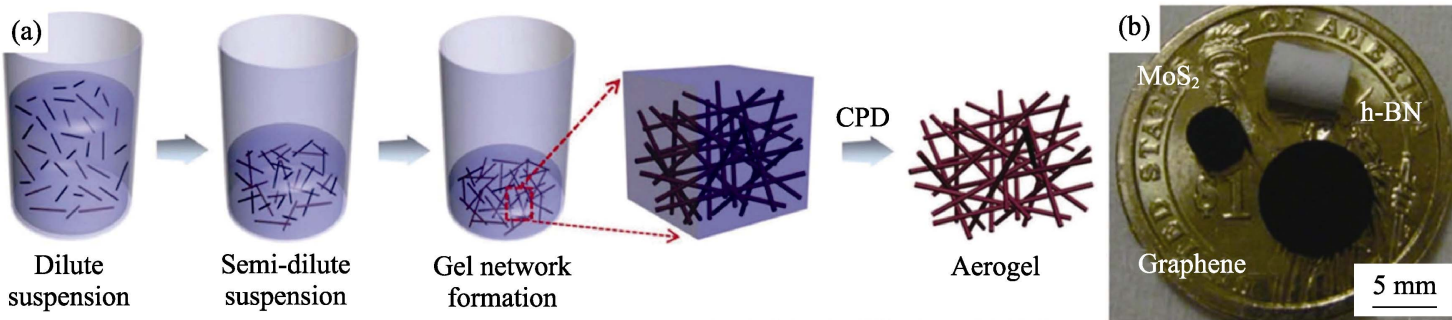

图 5 (a)超临界干燥法制备 $\mathrm{BN}$ 气凝胶流程示意图, (b)BN 气凝胶、 $\mathrm{MoS}_{2}$ 气凝胶和 $\mathrm{GA}$ 照片 ${ }^{[40]}$

Fig. 5 (a) Schematic representation of aerogel production by a critical point drying method; (b) Picture of the as-obtained $\mathrm{BN}$ aerogels, $\mathrm{MoS}_{2}$ aerogels and $\mathrm{GA}^{[40]}$
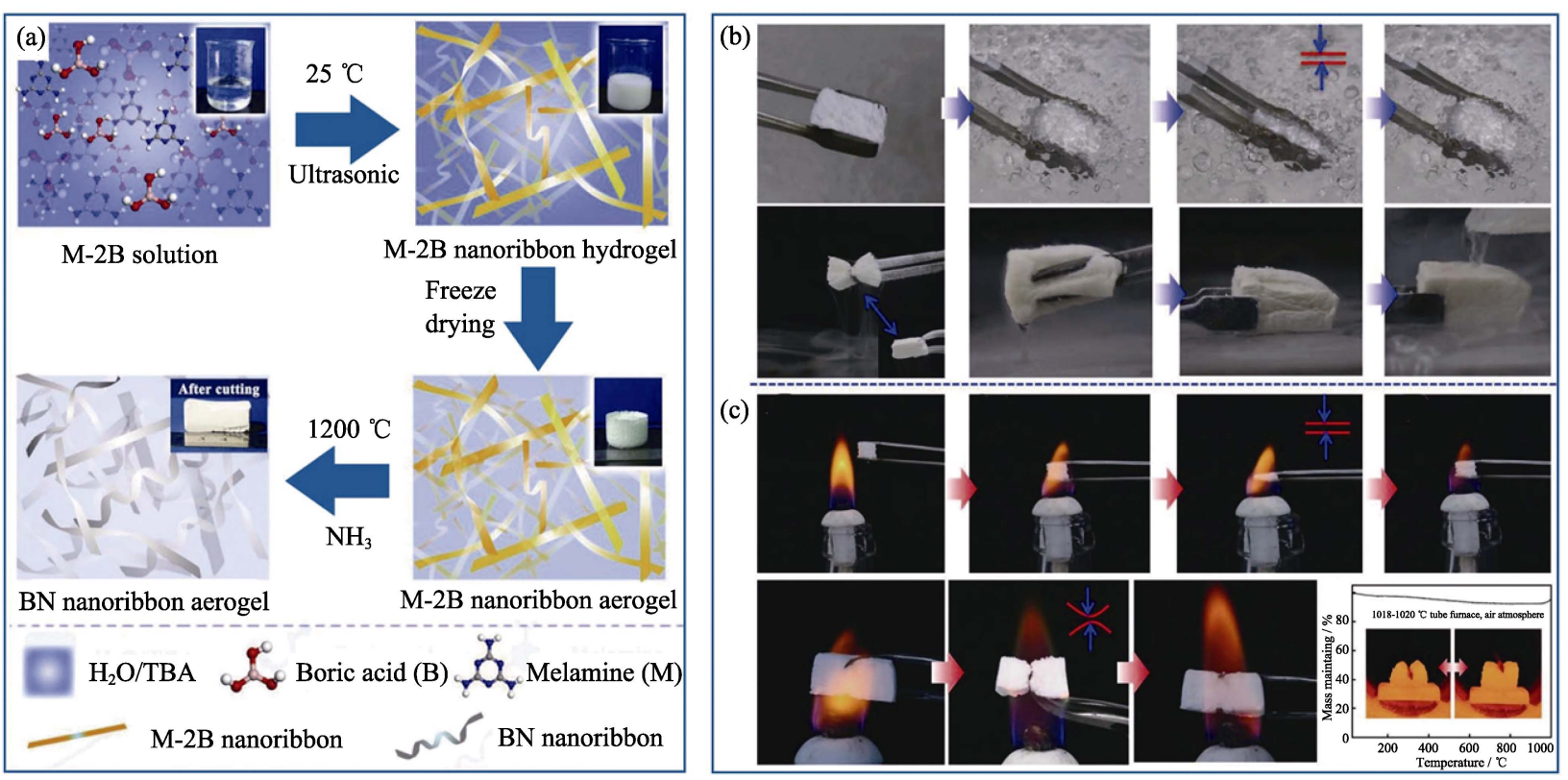

图 6 (a)冷冻干燥法制备纳米带状 $\mathrm{BN}$ 气凝胶流程示意图, $(\mathrm{b}, \mathrm{c}) \mathrm{BN}$ 纳米带气凝胶在液氮和火焰中的柔韧性 ${ }^{[41]}$

Fig. 6 (a) Schematic illustration of the freeze-drying method for preparing nano-ribbon BN aerogels; $(b, c)$ The flexibility of BN nano-ribbon aerogels in liquid nitrogen and flame ${ }^{[41]}$ 
备 h-BN 层和 $\mathrm{G}$ 的分散体，通过混合后得到 h-BN/G 块体。这些范德华堆叠的混合固体材料显示出与其 起始母体层明显不同的电学、机械和光学性质, 但 是这种材料由于孔隙率太低, 仍不属于气凝胶范 畴。2017 年, $\mathrm{Li}$ 等 ${ }^{[43]}$ 首次报道了无化学交联剂法制 备还原氧化石墨烯( $(\mathrm{GO}) / \mathrm{BN}$ 复合气凝胶(图 7)。研 究人员直接对混合均匀的 $\mathrm{rGO}$ 纳米片和 $\mathrm{BN}$ 纳米片 进行冷冻干燥, 在 $300{ }^{\circ} \mathrm{C}$ 下氢气还原得到密度仅为 $3.6 \mathrm{mg} \cdot \mathrm{cm}^{-3}$ 的复合气凝胶。均匀分布的 $\mathrm{BN}$ 纳米片 既阻止了 $\mathrm{rGO}$ 的团聚, 也促使其形成网状结构, 使 气凝胶在制备过程中没有明显的体积收缩。无交联 剂自组装法制备过程简单, 并且可得到任意尺寸和 形状的气凝胶, 有望用于大规模制备气凝胶。

\section{4 无模板法}

无模板法是在不借助外界模板的情况下，通过 $\mathrm{B}$ 源和 $\mathrm{N}$ 源直接发生化学反应制备多孔气凝胶的方 法。2010 年, Rao 课题组以硼酸和尿素为原料, 直接 通过二者反应制备出 $\mathrm{BN}$ 气凝胶, 经通过调整原料的 比例和优化反应条件, 得到比表面积为 $927 \mathrm{~m}^{2} \cdot \mathrm{g}^{-1}$ 的多孔气凝胶 ${ }^{[4]}$ 。研究者也尝试过用其他方法制备 $\mathrm{BN}$ 气凝胶, 如高压蒸汽法、热聚合 $\mathrm{BH}_{3} \mathrm{NH}_{3}$ 法等 ${ }^{[45-47]}$, 但是所得气凝胶的比表面积和孔体积都不尽人意。 近几年, 随着研究的深入, 无模板法制备 $\mathrm{BN}$ 气凝 胶取得了突破性进展。Weng 等 ${ }^{[21]}$ 以硼酸和双氰胺为 先驱体, 制备出比表面积高达 $1900 \mathrm{~m}^{2} \cdot \mathrm{g}^{-1}$ 的 $\mathrm{BN}$ 气 凝胶。有趣的是, 在透射扫描电镜下并不能观察到
孔的存在(图 8(a)), 通过高分辨透射电镜进一步观 察, 可以发现晶格存在错位(图 8(b,c)), 这是因为气 凝胶的孔径仅有 $1 \mathrm{~nm}$, 并且仅存在于 $\mathrm{BN}$ 层间的“褶 皱”中(如 P1、P2 所示)。类似的, Lei 等 ${ }^{[48]}$ 利用嗍酸盐酸胍为先驱体合成出了比表面积为 $1425 \mathrm{~m}^{2} \cdot \mathrm{g}^{-1}$ 的 $\mathrm{BN}$ 气凝胶。总之, 相比于模板法, 无模板法不受 模板的空间和形态限制, 是制备高比表面积 BN 气 凝胶的有效方法。

\section{$2 \mathrm{BN}$ 气凝胶的应用}

近年来, GA 凭借自身优异的物理化学性质, 以 及在能量储存、环保、催化等领域的应用潜力 ${ }^{[49-50]}$, 成为研究热点之一。而作为 $\mathrm{GA}$ 的等电子体, $\mathrm{BN}$ 气 凝胶不仅继承了 $\mathrm{BN}$ 的一系列优点，如机械强度好、 高润滑性、质轻、生物相容性好等, 而且弥补了部 分不足。BN 自身的性能优势以及多孔气凝胶的结构 特点使 BN 气凝胶具有高比表面积、良好的抗氧化 性和化学稳定性, 从而在气体吸附、催化、污水净 化、导热/隔热等诸多领域有更为广泛的应用前景。

\section{1 气体吸附}

氢能作为一种储量丰富、来源广泛、能量密度 高的绿色能源, 引起了人们的高度重视, 而氢能的 储运则是氢能应用的关键。研究表明, $\mathrm{H}_{2}$ 分子在 $B N$ 表面的结合能比在碳上的结合能高 $40 \%$, 因此 BN 气凝胶有望用作储氢材料 ${ }^{[11]}$ 。上文提到, Weng 等 ${ }^{[21]}$

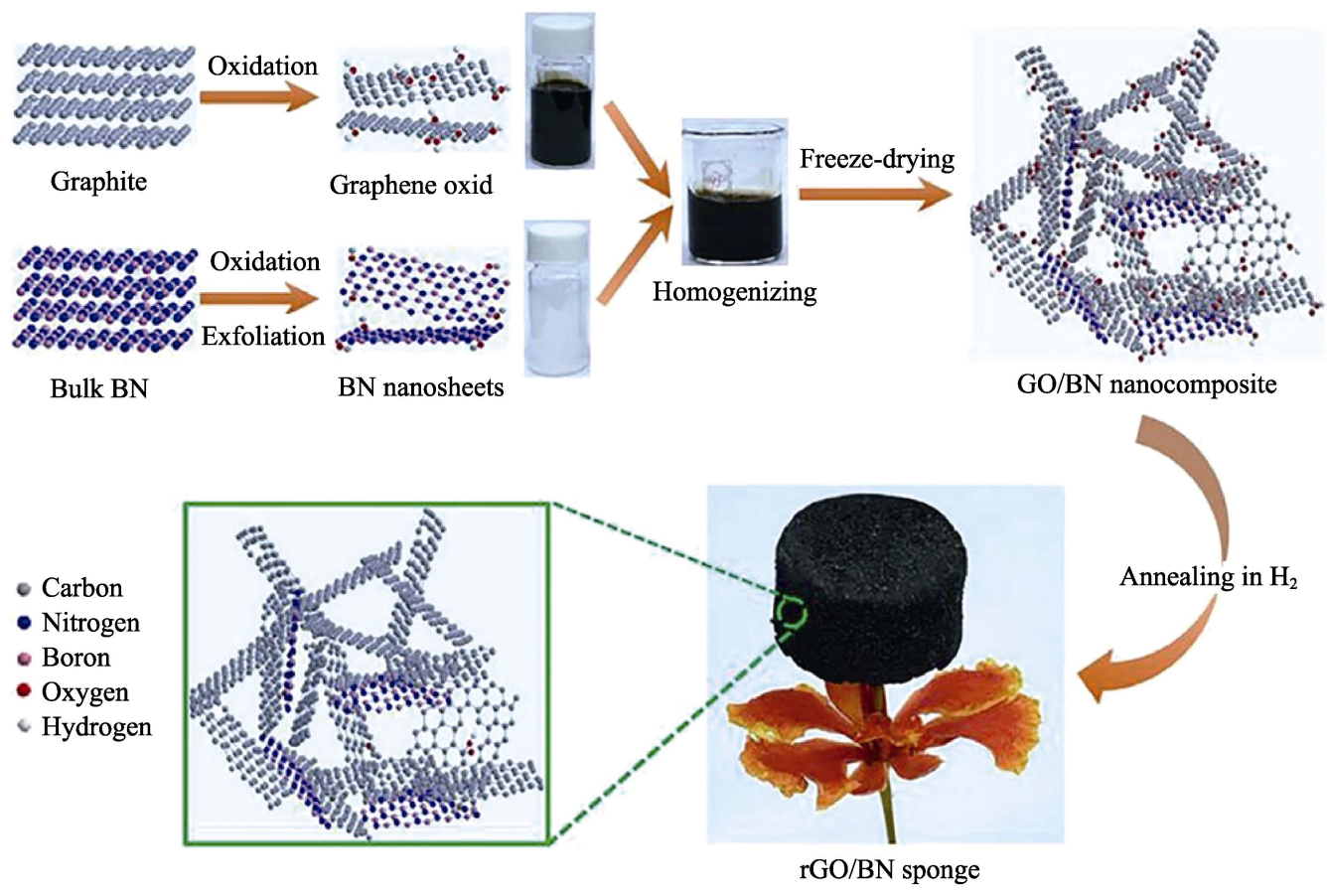

图 7 无交联剂法制备 $\mathrm{rGO} / \mathrm{BN}$ 复合气凝胶的流程示意图 ${ }^{[43]}$

Fig. 7 Schematic illustration of the preparation procedure of crosslinking-free $\mathrm{rGO} / \mathrm{BN}$ composite aerogels ${ }^{[43]}$ 

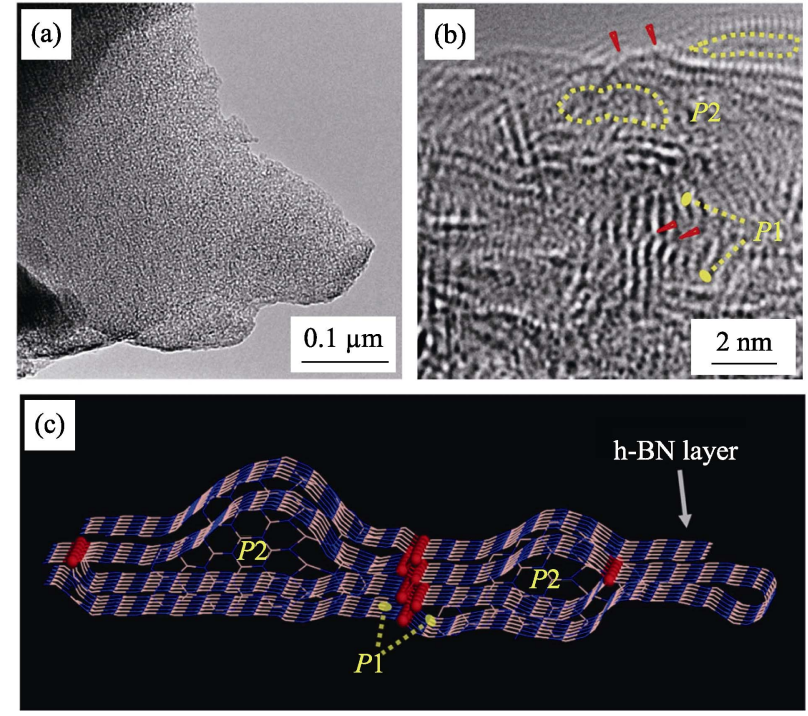

图 $8 \mathrm{BN}$ 气凝胶的 $(\mathrm{a}, \mathrm{b}) \mathrm{TEM}$ 照片及(c)微观结构示意图 ${ }^{[21]}$

Fig. 8 (a,b) TEM images of BN aerogels and (c) schematic illustration of microstructure ${ }^{[21]}$

采用无模板法合成出孔隙率为 $1.07 \mathrm{~cm} \cdot \mathrm{g}^{3}$ 的高比 表面积 $\mathrm{BN}$ 气凝胶 $\left(1900 \mathrm{~m}^{2} \cdot \mathrm{g}^{-1}\right)$, 这种气凝胶在 $-196{ }^{\circ} \mathrm{C} 、 1 \mathrm{MPa}$ 的条件下, 对氢气的吸收能力高达 $2.57 \%$ 。多孔 $\mathrm{BN}$ 气凝胶不仅可以储氢，而且对二氧 化碳、丙烷等气体展现出良好的吸附能力。近期, Kutty 等 ${ }^{[28]}$ 利用原子取代法制备的 $\mathrm{BN}$ 气凝胶对二氧
化碳气体表现出较高的选择性，无论在室温(298 K) 和低温 $(273 \mathrm{~K})$ 条件下, 对二氧化碳的吸收量都远高 于氮气(如图 9(a,b))。相似的, Anna 等 ${ }^{[52]}$ 通过在 BN 气凝胶上负载 Pt 纳米晶制备出高性能丙烷气体传 感器(图 9(c))。该传感器不仅在 $500{ }^{\circ} \mathrm{C}$ 下有良好的 稳定性，而且具有较快的响应/恢复速率(1.35 $\mathrm{s}$ 和 $0.6 \mathrm{~s}$ )(图 9(d)), 这是因为 $\mathrm{BN}$ 的高热导率可以有效 防止热点堆积和纳米粒子的烧结。

\section{2 催化}

BN 良好的化学稳定性和抗氧化性使其在高温 和氧化条件下表现出优于传统催化剂载体的性能。 此外， BN 气凝胶的多孔网状结构有利于反应物的 扩散, 超高的比表面积可以最大程度地负载金属活 性组分, 进而提高催化性能 ${ }^{[53-54]}$ 。虽然甲醇燃料电 池被认为是很有前途的“绿色”便携式发电机，但它 们的进一步发展很大程度上受限于目前 Pt 基阳极 催化剂的高成本和低催化活性。近期, $\mathrm{Li}$ 等 ${ }^{[55]}$ 提出 了一种“自下而上”的大规模制造超细 Pt 纳米颗粒/ $\mathrm{BN}$-石墨烯掺杂气凝胶 $(\mathrm{Pt} / \mathrm{BN}-\mathrm{GA})$ 的方法。 $\mathrm{Pt} / \mathrm{BN}$ GA 催化剂具有的三维交联多孔网络, 高比表面积 $\left(369.2 \mathrm{~m}^{2} \cdot \mathrm{g}^{-1}\right)$, 众多 $\mathrm{B}$ 和 $\mathrm{N}$ 活性位点, 均匀分散的 $\mathrm{Pt}$ 纳米颗粒和良好的导电性，构成了甲醇燃料电池 理想的阳极催化体系(图 10(a))。因此，相比于传统
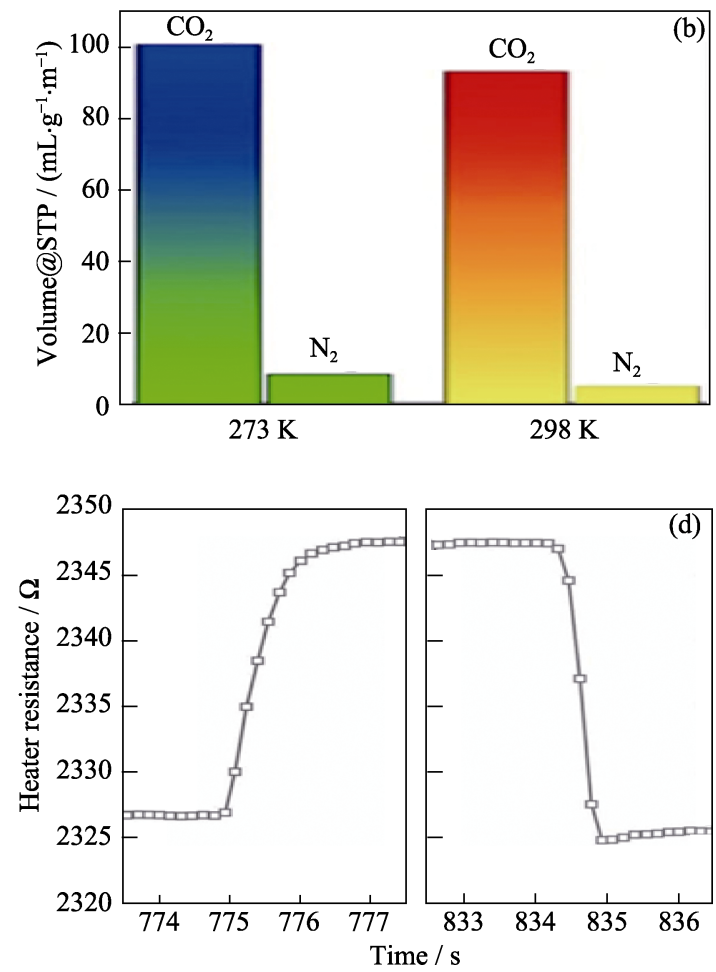

图 9 (a)在 273 和 $298 \mathrm{~K}$ 下, $\mathrm{BN}$ 气凝胶对 $\mathrm{CO}_{2}$ 和 $\mathrm{N}_{2}$ 的吸收量及(b)相应的柱状图 ${ }^{[28]}$, (c)Pt 纳米晶/BN 气凝胶的 SEM 照片, (d)Pt 纳米晶/BN 气凝胶对丙烷的响应/恢复曲线 ${ }^{[52]}$

Fig. 9 (a) The absorption of $\mathrm{CO}_{2}$ and $\mathrm{N}_{2}$ at 273 and $298 \mathrm{~K}$ by BN aerogel and (b) corresponding histograms ${ }^{[28]}$; (c) SEM image of Pt nanocrystals/BN aerogel; (d) Response/recovery curve of Pt nanocrystal/BN aerogel towards propane ${ }^{[52]}$ 

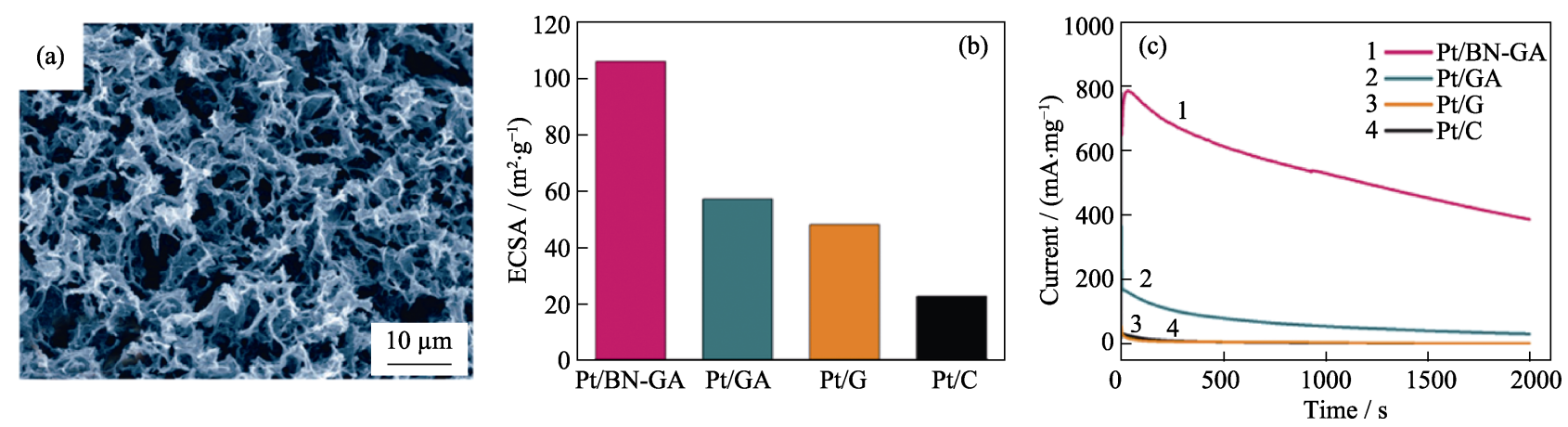

图 10 (a)Pt/BN-GA 催化剂的 SEM 照片 ${ }^{[55]}$, (b)Pt/BN-GA、Pt/GA、Pt/G 和 Pt/C 的 ECSA 对比图和(c)电流-时间曲线 ${ }^{[57]}$

Fig. 10 (a) SEM images of Pt/BN-GA catalyst ${ }^{[55]}$; (b) ECSA comparison chart of $\mathrm{Pt} / \mathrm{BN}-\mathrm{GA}, \mathrm{Pt} / \mathrm{GA}, \mathrm{Pt} / \mathrm{G}$ and $\mathrm{Pt} / \mathrm{C}$ and (c) corresponding current-time curves ${ }^{[57]}$

的 $\mathrm{Pt} /$ 炭黑 $(\mathrm{Pt} / \mathrm{C}) 、 \mathrm{Pt} / \mathrm{G}$ 和 $\mathrm{Pt} / \mathrm{GA}$ 催化剂, $\mathrm{Pt} / \mathrm{BN}-\mathrm{GA}$ 催化剂的催化活性、耐久性和强抗毒性得到显著提 高。实验表明, Pt/BN-GA 催化剂的电化学活性面积 (ECSA) 高达 $106.0 \mathrm{~m}^{2} \cdot \mathrm{g}^{-1}$, 分别是 $\mathrm{Pt} / \mathrm{GA} 、 \mathrm{Pt} / \mathrm{G}$ 和 $\mathrm{Pt} / \mathrm{C}$ 催化剂的 1.9、2.2 和 4.7 倍(图 10(b))。经测试, $\mathrm{Pt} / \mathrm{BN}-\mathrm{GA}$ 催化剂的初始电流在 $2 \times 10^{3} \mathrm{~s}$ 内仅下降了 39\%(图 10(c)), 远低于 $\mathrm{Pt} / \mathrm{GA}(\sim 78 \%) 、 \mathrm{Pt} / \mathrm{G}(\sim 93 \%)$ 和 $\mathrm{Pt} / \mathrm{C}(95 \%)$ 。

另外, 人们普遍认为宽带隙和化学惰性的 h-BN 不适合用于光催化和光伏转换材料。然而, Weng 等 ${ }^{[56]}$ 通过结构设计使 $\mathrm{BN}$ 气凝胶羟基功能化 的(002)晶面充分暴露, 在紫外和可见光范围内表现 出广谱吸收, 与锐钛矿型 $\mathrm{TiO}_{2}$ 纳米粒子杂交后, 制 备的 $\mathrm{TiO}_{2} / \mathrm{BN}$ 气凝胶在 $4420 \mathrm{~nm}$ 可见光下对乙酸和 染料(结晶紫)降解有较强的光催化作用, 是有机化 合物氧化降解的有效光催化剂。值得注意的是, BN 气凝胶自身也可以作为催化剂。Chen 等 ${ }^{[57]}$ 制备出 $\mathrm{BN} /$ 石墨烯杂化气凝胶 $(\mathrm{BN} / \mathrm{GA})$, 可用作高效的双 效氧电催化剂。 $\mathrm{BN} / \mathrm{GA}$ 不仅表现出与商业 $\mathrm{Pt} / \mathrm{C}$ 相 似的氧化还原反应活性, 而且与商用 $\mathrm{Pt} / \mathrm{C}$ 相比, 还 具有更优异的稳定性和甲醇耐受性。同时, BN/GA 表现出的析氧反应活性使其有望作为锌一空气电池 装置的阴极。

\section{3 污水净化}

研究表明, 吸附效率与吸附剂的孔径尺寸、孔 隙率和比表面积密切相关 ${ }^{[58]}$ 。BN 气凝胶中微小而 均匀分布的孔隙不仅赋予 $\mathrm{BN}$ 气凝胶超高的比表面 积，使吸附位点充分暴露，而且有效减小了被吸附 物由气凝胶表面进入内部的阻力, 更有利于吸附过 程的持续进行。此外, $\mathrm{BN}$ 气凝胶良好的疏水亲油性 保证了吸附水中有机溶剂的能力, 并呈现出经济和 高效的特点。 $\mathrm{Li}$ 等 ${ }^{[59]}$ 制备的 $\mathrm{rGO} / \mathrm{BN}$ 气凝胶不仅具 有卓越的机械性能还具有优异的疏水吸油能力, 吸 油量达到自身重量的 170 倍, 是用于污水净化的良
好材料(图 11)。Zhao 等 ${ }^{[60]}$ 和 Pham 等 ${ }^{[61]}$ 制备的 BN 气凝胶也表现出优异的吸油能力, 分别可吸附自身 重量的 150 倍和 190 倍的有机污染物。更重要的是, BN 气凝胶具有良好的热稳定性和机械性能, 吸附 了油污的 $\mathrm{BN}$ 气凝胶可以通过简单挤压和燃烧的 方式除去污染物，从而实现低成本循环利用。Song 等 ${ }^{[62]}$ 采用 CVD 法制备出的 $\mathrm{BN}$ 气凝胶可在 $5 \mathrm{~s}$ 之内 吸收自身重量 160 倍的油, 饱和的 BN 气凝胶在空 气中灼烧后可循环使用, 并且没有明显的性能下 降。除此之外, $\mathrm{BN}$ 气凝胶还可用于吸附污水中的重 金属离子, Xue 等 ${ }^{[63]}$ 制备的气凝胶在水中对 $\mathrm{Cd}^{2+}$ 的 吸附能力达到 $561 \mathrm{mg} \cdot \mathrm{g}^{-1}$ 。

\section{4 导热与隔热}

BN 与其他材料复合后，由于自身的高热导率， 可大幅度提高复合材料的导热性能; 另外, 通过结 构设计, $\mathrm{BN}$ 气凝胶因独特的纳米多孔网络结构而具 有高孔隙率、高比表面积、低密度及低热导率等性 质，也是一种理想的隔热材料。石墨烯气凝胶是一 种很有潜力的导热材料, 但其高孔隙率、低密度和 各向同性的结构阻碍了其热导率的进一步提高。An 等 ${ }^{[64]}$ 通过水热处理 $\mathrm{GO}$ 和 $\mathrm{BN}$ 纳米片的悬浮液, 制 备出具有长程有序结构和中等密度的高度各向异性 $\mathrm{rGO} / \mathrm{BN}$ 混合气凝胶(图 12)。所得复合材料具有 $11.01 \mathrm{~W} \cdot \mathrm{m}^{-1} \cdot \mathrm{K}^{-1}$ 的超高平面导热率，相比于纯石墨 烯气凝胶，导热率提高了 $277 \%$ 。另一方面, $\mathrm{Xu}$ 等 ${ }^{[26]}$ 采用多尺度结构化设计和石墨烯气凝胶模板, 合成 了兼具良好机械和低热导率的层状双壁结构 BN 气 凝胶(图 13)。层状双壁结构将气凝胶分成微小的单 元，有效降低了空气对流，小尺寸的晶粒和中空的 双壁结构减少了固体传导，大幅抑制了声子散射， 从而实现低于空气的超低热导率 $\left(0.02 \mathrm{~W} \cdot \mathrm{m}^{-1} \cdot \mathrm{K}^{-1}\right)$ 。 在高温热振测试中, 这类材料表现出优异的热稳定 性，机械强度损失不到 $1 \%$ 。因此，这种材料在航空 航天隔热领域有广阔的发展前景。 
(a)

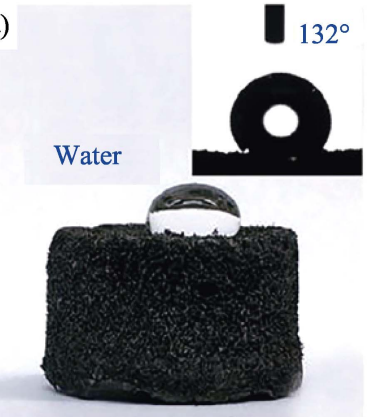

(b)

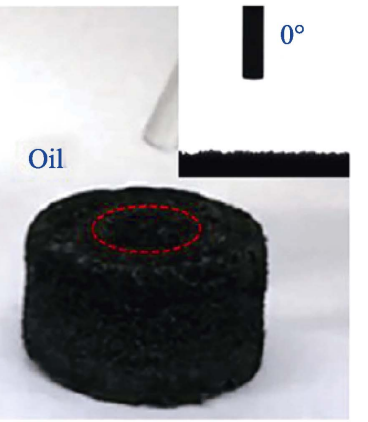

(c) Oil stained with

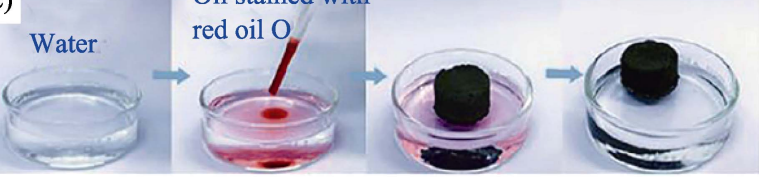

(d)
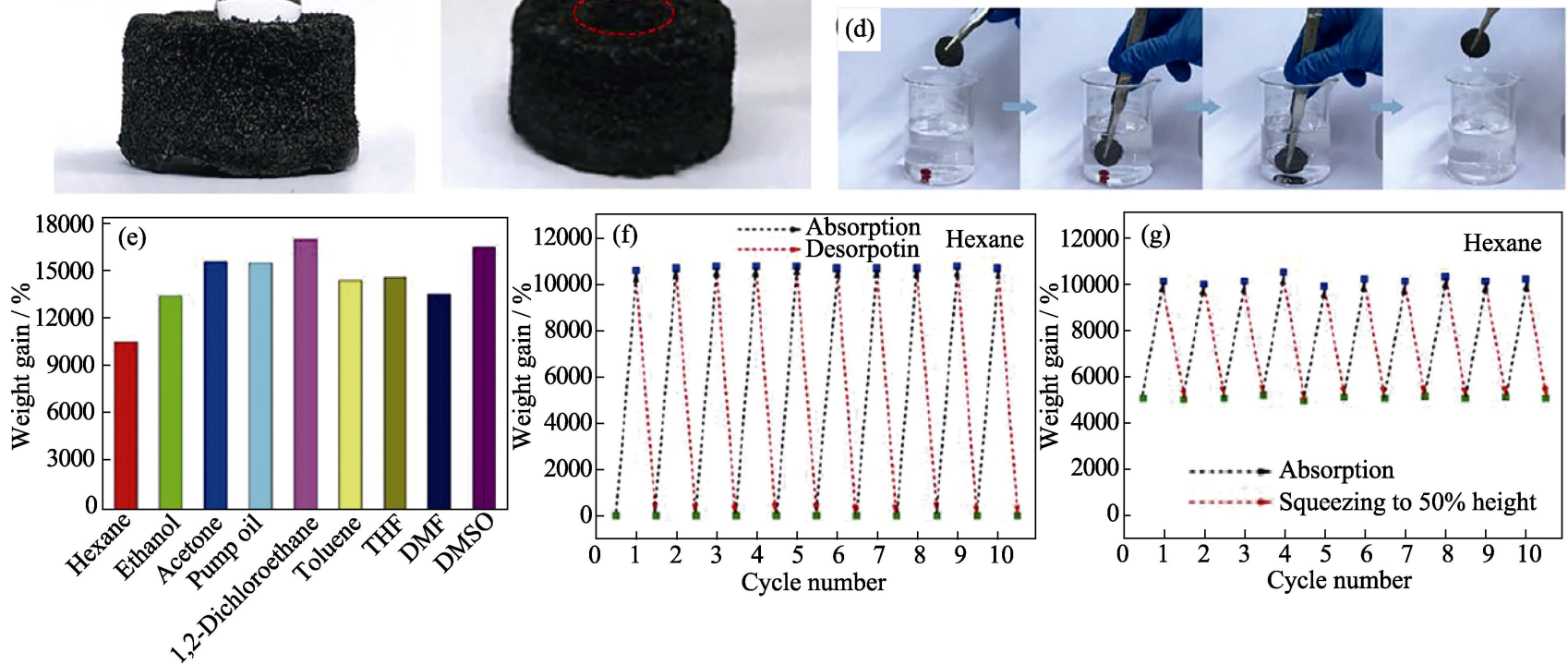

图 $11(\mathrm{a} \sim \mathrm{d}) \mathrm{rGO} / \mathrm{BN}$ 气凝胶的润湿行为和吸油能力, (e) $\mathrm{rGO} / \mathrm{BN}$ 气凝胶吸收不同有机液体的能力, (f) $\mathrm{rGO} / \mathrm{BN}$ 气凝胶反复 吸收己烷并在热处理 $\left(85^{\circ} \mathrm{C}\right)$ 下释放其蒸汽的循环曲线, $(\mathrm{g}) \mathrm{rGO} / \mathrm{BN}$ 气凝胶在反复吸收-挤压下吸收己烷的循环曲线 ${ }^{[59]}$

Fig. 11 (a-d) The Wetting behaviour and oil absorption capacity of $\mathrm{rGO} / \mathrm{BN}$ sponge; (e) The ability of $\mathrm{rGO} / \mathrm{BN}$ sponge to absorb different organic liquids; (f) The $\mathrm{rGO} / \mathrm{BN}$ sponge repetitively absorbed hexane and released its vapour under heat treatment $\left(85^{\circ} \mathrm{C}\right)$; $(\mathrm{g})$ Recyclability of the $\mathrm{rGO} / \mathrm{BN}$ sponge for absorption of hexane under absorption-squeezing cycles ${ }^{[59]}$

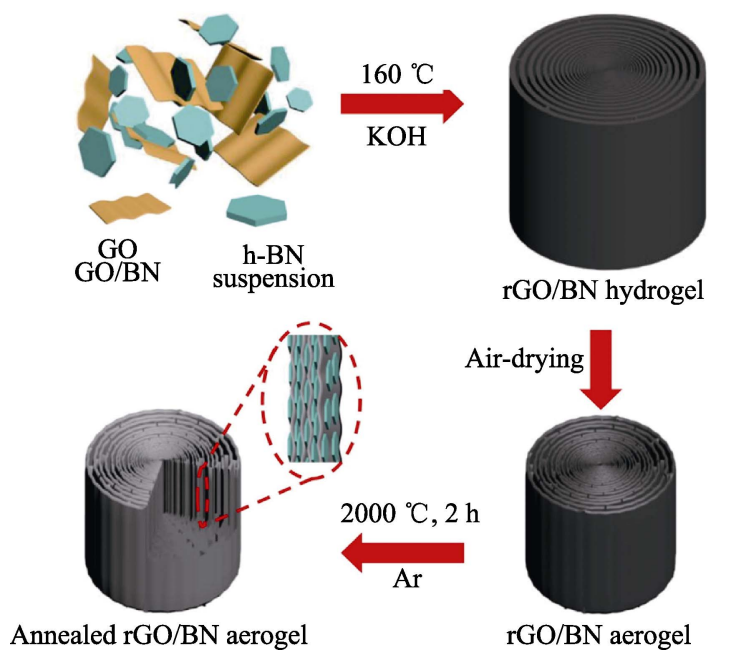

图 $12 \mathrm{rGO} / \mathrm{BN}$ 混合气凝胶的制备流程示意图 ${ }^{[64]}$

Fig. 12 Schematic illustrating the fabrication of graphene/BN hybrid aerogels ${ }^{[64]}$

凭借自身优异的性能, BN 气凝胶在其他重要领 域也有很多潜在的应用。例如, 新兴的化学功能化 方法为 BN 气凝胶未来的界面改性提供了良好的机 会。可以将其他组分化学键合到 $\mathrm{BN}$ 气凝胶表面，从 而开发高效耐用的纳米催化剂或用于调整复合材料 的带结构以改善光生空穴和电子分离。此外，研究 表明, BN 纳米结构比碳材料具有更好的生物相容性
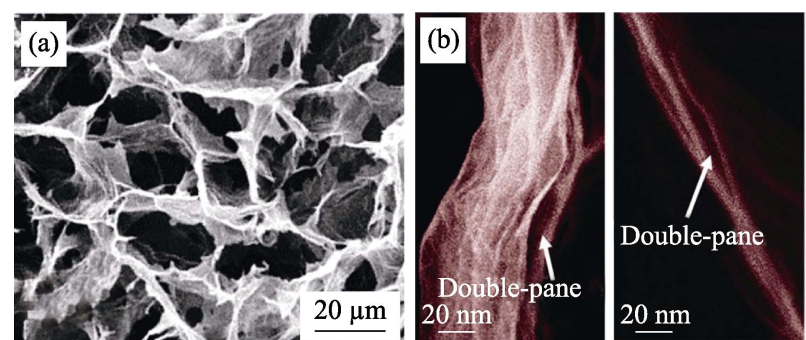

图 13 层状双壁结构 $\mathrm{BN}$ 气凝胶的 SEM 照片 ${ }^{[26]}$

Fig. 13 SEM images of the double-pane wall structure of BN aerogels ${ }^{[26]}$

(a) hBNAG; (b) Double-pane wall structure of hBNAGs. Scale bars, $20 \mathrm{~nm}$

和更低的细胞毒性 ${ }^{[65-66]}$ 。Weng 等 ${ }^{[67]}$ 报道了一种简单 的热取代方法，制造出高羟基化水溶性 BN 气凝胶。 这些着基化的 $\mathrm{BN}$ 气凝胶具有生物相容性，并且可 以有效地加载抗癌药物(例如多柔比星, DOX)直至 超过其自身重量三倍。加载在这种 BN 载体上相同 或甚至更少的药物可表现出比游离药物更强的抑制 $\mathrm{LNCaP}$ 癌细胞活力的效力, 是一种良好的药物载体。

\section{3 结束语}

BN 气凝胶具有低密度、孔隙率高、高比表面积、 良好的抗氧化性和化学稳定性等优异性能，在气体 
吸附、催化、污水净化、导热/隔热等领域极具应用 前景。但是相比于碳材料, 其在相关领域的研究还不 够充分, 因此拓宽其应用领域是值得重视的课题。一 个可能的方案就是通过化学修饰或掺杂改变 $\mathrm{BN}$ 的 组分, 进而调控其性质, 但 BN 的高化学惰性阻碍了 对其进行后处理和改性。迄今为止, 已经建立了各种 用于 BN 功能化的后合成方法, 然而, 这些策略的范 围和效率仍然很少令人满意。另外，如何精准控制 $\mathrm{BN}$ 气凝胶的孔结构, 包括孔径尺寸和孔隙率, 仍然 是一个挑战。除了发展合成方法, 设计精确的分析技 术表征 BN 纳米结构也是至关重要的。例如, 尖端的 高分辨率透射电子显微镜是一种在空间和化学上解 析单个原子的有力工具, 这项技术会为 $\mathrm{BN}$ 功能化研 究提供有价值的信息，比如精确测定 BN 表面的功能 化位置, 形成的键的几何形状和性质等。然而, 这个 技术仍然具有挑战性, 因为 $\mathrm{B} 、 \mathrm{C}$ 和 $\mathrm{N}$ 原子通常在显 微成像下显示出接近的原子尺寸和对比度, 难以直 接分辨。

考虑到上述限制，研究人员应该着力于开发更 有效和经济的 $\mathrm{BN}$ 气凝胶合成和功能化策略, 使其满 足未来高性能生物、热防护、电化学等装置的应用 需求。我们相信, 随着研究的深入, $\mathrm{BN}$ 气凝胶将呈现 出更为广阔的应用前景。

\section{参考文献:}

[1] 冯伟. 不同祄底上氮化硼薄膜制备与场发射性质研究. 长春: 吉林大学硕士学位论文, 2004

[2] DU M, LI Y, ZHANG G R, et al. Progress in preparation and application of boron nitride nanosheets. Inorganic Chemicals Industry, 2019, 51(2): 8 .

[3] WANG G Z. Summarization about the peculiarities of cBN. Jewellery Science and Technology, 2005, 17(5): 41-45.

[4] WANG G Z. Synthesis and structure transformation of wBN under HPHT. Inorganic Chemicals Industry, 2006, 18(4): 21-24.

[5] YANG Y P, LI B, ZHANG C Y, et al. The Morphology, synthesis, properties, and applications of graphene-like two-dimensional h-BN nanomaterials. Materials Review, 2016, 30(11): 143-148.

[6] PAKDEL A, BANDO Y, GOLBERG D. Nano boron nitride flatland. Chemistry Society Reviews, 2014, 43(3): 934-959.

[7] LIN Y, CONNELL J W. Advances in 2D boron nitride nanostructures: nanosheets, nanoribbons, nanosheets, and hybrids with graphene. Nanoscale, 2012, 4(22): 6908-6939.

[8] ZHI C Y, BANDO Y, TANG C C, et al. Large-scale fabrication of boron nitride nanosheets and their utilization in polymeric composites with improved thermal and mechanical properties. Advanced Materials, 2009, 21(28): 2889-2893.

[9] ZHAO Y, WU X J, YANG J L, et al. Oxidation of a two-dimensional hexagonal boron nitride monolayer: a first-principles study. Physical Chemistry Chemical Physics, 2012, 14(16): 5545-5550.

[10] WATANABE K, TANIGUCHI T, KANDA H. Direct-bandgap properties and evidence for ultraviolet lasing of hexagonal boron nitride single crystal. Nature Materials, 2004, 3(6): 404-409.

[11] LI L H, CHEN Y. Atomically thin boron nitride: unique properties and applications. Advanced Functianal Materials, 2016, 26(16): 2594-2608.

[12] DUAN X M, YANG J H, WANG Y J, et al. Research and application progress of hexagonal boron nitride (h-BN) based composite ceramics. Materials China, 2015, 34(10): 770-782.

[13] JIANG X F, WENG Q, WANG X B, et a1. Recent progress on fabrications and applications of boron nitride nanomaterials: a review. Journal of Materials Science \& Technology, 2015, 31(6): 589-598.

[14] NICOLA H U S. Aerogels-airy materials: chemistry, structure, and properties. Angewandte Chemie International Edition, 1998, 37(1/2): $22-45$.

[15] XIAO Y Y, JIANG Y G, FENG J Z, et al. Research progress of polyurethane based aerogel insulation materials. Materials Review, 2018, 32(1): 449-453.

[16] LUO Y, JIANG Y G, FENG J Z, et al. Progress on the preparation of $\mathrm{SiO}_{2}$ aerogel composites by ambient pressure drying technique. Materials Review, 2018, 32(5): 780-787.

[17] WATANABE K, TANIGUCHI T, KANDA H. Direct-bandgap properties and evidence for ultraviolet lasing of hexagonal boron nitride single crystal. Nature Materials, 2004, 3(6): 404.

[18] MENG Y, CHEN J J, LIANG W X, et al. Progress in catalysis of hexagonal boron nitride and boron nitride nanosheets. Journal of Southwest University for Nationalities (Natural Science Edition), 2015, 41(3): 331-337.

[19] LI J, LI L J, GAO Y F, et al. Preparation of nanomaterials employing template method. Materials Review, 2011(s2): 5-9.

[20] RUAN X, DONG L, YU J, et al. The progress of nanomaterials prepared in the presence of soft template. Materials Review, 2012, 26(1): 56-60.

[21] WENG Q, WANG X, BANDO Y, et al. One-step template-free synthesis of highly porous boron nitride microsponges for hydrogen storage. Advanced Energy Materials, 2014, 4(7): 130-152.

[22] DAI J, WU X, YANG J, et al. Unusual metallic microporous boron nitride networks. Journal of Physical Chemistry Letters, 2013, 4(20): 3484-3488.

[23] DAI J, WU X, YANG J, et al. Porous boron nitride with tunable pore size. Journal of Physical Chemistry Letters, 2014, 5(2): 393-398.

[24] HAN W Q, BRUTCHEY R, TILLEY T D, et al. Activated boron nitride derived from activated carbon. Nano Letters, 2004, 4(1): 173-176.

[25] RUSHTON B, MOKAYA R. Mesoporous boron nitride and boronnitride-carbon materials from mesoporous silica templates. Journal of Materials Chemistry, 2007, 18(2): 235-241.

[26] XU X, ZHANG Q Q, HAO M L, et al. Double-negative-index ceramic aerogels for thermal superinsulation. Science, 2019, 363: 723-727.

[27] ZHANG Q, XU X, LIN D, et al. Hyperbolically patterned 3D graphene metamaterial with negative poisson ratio and superelasticity. Advanced Materials, 2016, 28(11): 2229-2237.

[28] KUTTY R G, SREEJITH S, KONG X, et al. A topologically substituted boron nitride hybrid aerogel for highly selective $\mathrm{CO}_{2}$ uptake. Nano Research, 2018, 11(12): 6325-6335.

[29] YIN J, LI X, ZHOU J, et al. Ultralight three-dimensional boron nitride foam with ultralow perm ittivity and superelasticity. Nano Letters, 2013, 13(7): 3232-3236.

[30] ALAUZUN J G, NGURN S, BRUN N, et al. Novel monolith-type boron nitride hierarchical foams obtained through integrative chemistry. Journal of Materials Chemistry, 2011, 21(36): 14025-14030.

[31] HUANG Y F, LIU H E, WANG Z Y, et al. Preparation of graphene aerogels with soft templates and their oil adsorption mechanism from water. Journal of Chemical Engineering of Chinese Universities, 2012, 26(1): 56-60. 
[32] YAMAUCHI Y, KURODA K. Rational design of mesoporous metals and related nanomaterials by a soft-template approach. Chemistry - An Asian Journal, 2010, 3(4): 664-676.

[33] WANG H, FAN G, ZHENG C, et al. Facile sodium alginate assisted assembly of $\mathrm{Ni} / \mathrm{Al}$ layered double hydroxide nanostructures. Industrial \& Engineering Chemistry Research, 2010, 49(6): 2759-2767.

[34] XIU R, DONG L, JING Y U, et al. The progress of nanomaterials prepared in the presence of soft template. Materials Review, 2012, 1: 56-60.

[35] BERNARD S, MIELE P. Nanostructured and architectured boron nitride from boron, nitrogen and hydrogen-containing molecular and polymeric precursors. Materials Today, 2014, 17(9): 443-450.

[36] MALENFANT P R L, WAN J, TAYLOR S T, et al. Self-assembly of an organic-inorganic block copolymer for nano-ordered ceramics. Nature Nanotechnology, 2007, 2(1): 43-46.

[37] QIAN X H, LIU H B, LI Y L. Self-assembly low dimensional inorganic/organic heterojunction nanomaterials. Chinese Science Bulletin, 2014(1): 1-14.

[38] KLOK H A, LECOMMANDOUX S. Supramolecular materials via block copolymer self-assembly. Advanced Materials, 2010, 13(16): 1217-1229.

[39] CIFERRI A. Assembling nano and macrostructures and the supramolecular liquid crystal. Progress in Polymer Science, 1995, 20(6): 1081-1120.

[40] JUNG S M, JUNG H Y, DRESSELHAUS M S, et al. A facile route for $3 \mathrm{D}$ aerogels from nanostructured $1 \mathrm{D}$ and $2 \mathrm{D}$ materials. Scientific Reports, 2013, 2(11): 849.

[41] LI G Y, ZHU M Y, GONG W B, et al. Boron nitride aerogels with super-flexibility ranging from liquid nitrogen temperature to $1000{ }^{\circ} \mathrm{C}$. Advanced Functional Materials, 2019, 29: 1900188.

[42] GAO G, GAO W, CANNUCCIA E, et al. Artificially stacked atomic layers: toward new van der waals solids. Nano Letters, 2012, 12(7): 3518-3525.

[43] LI H, LIN J, TAY R Y, et al. Multifunctional and highly compressive cross-linker-free sponge based on reduced graphene oxide and boron nitride nanosheets. Chemical Engineering Journal, 2017, 328: $825-833$.

[44] NAG A, RAIDONGIA K, HEMBRAM K P, et al. Graphene analogues of BN: novel synthesis and properties. ACS Nano, 2010, 4(3): $1539-1544$.

[45] MENG X, LUN N, QI Y, et al. Low-temperature synthesis of meshy boron nitride with a large surface area. European Journal of Inorganic Chemistry, 2010(20): 3174-3178.

[46] MENG X L, LUN N, QI Y X, et al. Simple synthesis of mesoporous boron nitride with strong cathodoluminescence emission. Journal of Solid State Chemistry, 2011, 184(4): 859-862.

[47] MENG W, LI M, XU L, et al. High yield synthesis of novel boron nitride submicro-boxes and their photocatalytic application under visible light irradiation. Catalysis Science \& Technology, 2011, 1(7): 1159

[48] LEI W, PORTEHAULT D, LIU D, et al. Porous boron nitride nanosheets for effective water cleaning. Nature Communications, 2016, 4(2): 1777.

[49] ZHANG Q, XIANG X, HUI L, et al. Mechanically robust honeycomb graphene aerogel multifunctional polymer composites. Carbon, 2015, 93: 659-670.

[50] SONG X, LIN L, RONG M, et al. Mussel-inspired, ultralight, multifunctional 3D nitrogen-doped graphene aerogel. Carbon, 2014, 80(1): 174-182.
[51] JHI S H, KWON Y K. Hydrogen adsorption on boron nitride nanotubes: a path to room temperature hydrogen storage. Physical Review B, 2004, 69(24): 245407.

[52] HARLEY-TROCHIMCZYK A, PHAM T, CHANG J, et al. Gas sensors: platinum nanoparticle loading of boron nitride aerogel and its use as a novel material for low-power catalytic gas sensing. Advanced Functional Materials, 2016, 26(3): 314.

[53] ZHENG M T, LIU Y L, GU Y L, et al. Synthesis and characterization of boron nitride sponges as a noveI support for metal nanoparticles. Science China Chemistry, 2008, 51(3): 205-210.

[54] PERDigON-MELON J A, AUROUX A, GUIMON C, et al. Micrometric BN powders used as catalyst support: influence of the precursor on the properties of the BN ceramic. Journal of Solid State Chemistry, 2004, 177(2): 609-615.

[55] LI M, JIANG Q, YAN M, et al. Three-dimensional boron- and nitrogen-codoped graphene aerogel supported pt nanoparticles as highly active electrocatalysts for methanol oxidation reaction. ACS Sustainable Chemistry \& Engineering, 2018, 6: 6644-6653.

[56] WENG Q, IDE Y, WANG X, et al. Design of BN porous sheets with richly exposed (002) plane edges and their application as $\mathrm{TiO}_{2}$ visible light sensitizer. Nano Energy, 2015, 16: 19-27.

[57] LI M, JIANG Q, YAN M, et al. Three-dimensional boron- and nitrogen-codoped graphene aerogel supported Pt nanoparticles as highly active electrocatalysts for methanol oxidation reaction. ACS Sustainable Chemistry \& Engineering, 2018, 6: 6644-6653.

[58] LIU D, LEI W, QIN S, et al. Template-free synthesis of functional 3D BN architecture for removal of dyes from water. Scientific Reports, 2014, 4: 4453.

[59] LI H, LIN J, TAY R Y, et al. Multifunctional and highly compressive cross-linker-free sponge based on reduced graphene oxide and boron nitride nanosheets. Chemical Engineering Journal, 2017, 328: $825-833$.

[60] ZHAO H, SONG X, ZENG H. 3D graphene foam scavengers: vesicant-assisted foaming boosts the gram-level yield and forms hierarchical pores for super-strong pollutant removal applications. NPG Asia Mater., 2015, 7(3): 168.

[61] PHAM T, GOLDSTEIN A P, LEWICK J P, et al. Nanoscale structure and superhydrophobicity of $\mathrm{sp}^{2}$-bonded boron nitride aerogels. Nanoscale, 2015, 7(23): 10449-10458.

[62] SONG Y, LI B, YANG S, et al. Ultralight boron nitride aerogels via template-assisted chemical vapor deposition. Scientific Reports, 2015, 5: 10337.

[63] XUE Y, DAI P, JIANG X, et al. Template-free synthesis of boron nitride foam-like porous monoliths and their high-end applications in water purification. Journal of Materials Chemistry A, 2016, 4(4): $1469-1478$.

[64] AN F, LI X, MIN P, et al. Highly anisotropic graphene/boron nitride hybrid aerogels with long-range ordered architecture and moderate density for highly thermally conductive composites. Carbon, 2018, 126: 119-127.

[65] NURUNNABI M, NAFIUJJAMAN M, LEE S J, et al. Preparation of ultrathin hexagonal boron nitride nanoplates for cancer cell imaging and neurotransmitter sensing. Chemical Communications, 2016, 52(36): 6146-6149.

[66] LU F S, WANG F, CAO L, et al. Hexagonal boron nitride nanomaterials: advances towards bio-applications. Nanoscience and Nanotechnology Letters, 2012, 4(10): 949-961.

[67] WENG Q, WANG B, WANG X, et al. Highly water-soluble, porous, and biocompatible boron nitrides for anticancer drug delivery. ACS Nano, 2014, 8(6): 6123-6130. 